\title{
A New Approach in Delivering Building Information Modeling Applications and Data to Remote Users over Cloud through Billboard Manager
}

\author{
Rajesh Bose \\ Ph.D. Scholar, Department of \\ Computer Science \& \\ Engineering, University of \\ Kalyani, West Bengal, India
}

\author{
Himadri Biswas \\ Assistant Professor in the \\ Department of Computer \\ Applications, Bengal College of \\ Engineering \& Technology, \\ Durgapur, West Bengal, India.
}

\author{
Debabrata Sarddar \\ Department of Computer \\ Science \& Engineering \\ University of Kalyani, Nadia, \\ West Bengal, India
}

\begin{abstract}
The business of civil construction and real estate development is booming of late in Asia and all over the world. Studies peg the estimated valuation of work-in-progress of such projects to be more than billions of dollars at current market rates. In order to rationalize costs and maximize productivity at every stage, companies are beginning to rely heavily on information technology. Cloud computing offers businesses an option to scale their investments in IT services based on their needs and demands. However, in order to remain competitive, construction companies need to have their engineers working at remote locations, to be up to date with the latest data and also empower them to feed in changes to design and construction in real time. In this paper, we present a proposed architecture which we call the Billboard Manager based BIM cloud environment. In this model, we use Citrix technologies to offer high-quality and reliable links to various BIM applications hosted either on private or on a hybrid cloud setup. Our proposed model has been designed to enable access to BIM applications from any geographic location over the Internet. In this paper, we also show how Billboard Manager can effectively manage storage and retrieval of data in conjunction with BIM applications.
\end{abstract}

\section{Keywords}

Cloud, Building Information Modeling, Billboard Manager, Citrix, SSL

\section{INTRODUCTION}

For a business to be effective, competitive and profitable, employees need to have secured and balanced access to crucial information. Cloud computing solutions can offer plans that allow any enterprise to rationalize its operating costs and maximize utilization of time and resources. Standard client-server architecture models enable employees to access information from locations that need to be fixed and planned well in advance. Cloud computing solutions, on the other hand, hand the crucial advantage of accessing data and information from any part of the globe.

Companies engaged in the business of construction are steadily moving from long-established practices of centralizing data storage to cloud computing models. This move has enabled construction companies to share information and collect data vital to business interests quickly and effectively. Geographical distances and remote locations no longer pose a serious problem in the day to day operations of construction companies. Construction workers and managers at sites far removed from cities and towns can be more productive by virtue of easily accessible data and applications that are hosted on cloud.

Constructions companies have begun transitioning to cloud computing models which are appropriate for their requirements for reasons directly related to scalability and cost-effectiveness. In addition to these reasons, construction companies are increasingly relying on cloud computing to deliver training to employees at remote locations. This hands a significant advantage in terms of flexibility, as well as multiplying effectiveness of available resources. Agility is a decisive factor if a business is to stay competitive. With intense competition in the construction sector, companies are increasingly focusing attention on training of their staff and empowering them with cutting-edge computer software tools. Cloud computing provides a dependable platform that helps construction companies move quickly in the targeted direction. New software tools and technologies can be rolled out as soon as possible. Wherever internet and/or wireless technologies are available at remote locations, new tools can be made available without deploying costly resources.

Cloud computing models can help construction companies focus on their core business without having to divert funds for procuring expensive computer hardware and software. Businesses can choose to rent infrastructure and services on an on-demand basis. This enables enterprises to rationalize their IT investment costs. Further, managers can choose to enable those service components and hire cloud computing platforms to support applications crucial to delivering large construction projects in association with other construction companies, consultants, architects, and clients.

\section{RELATED WORK}

The National Building Information Model Standard Project Committee based in the United States, defines BIM to be a digital representation of physical and functional characteristics of a facility [4]. BIM is further defined as a shared knowledge resource which forms a reliable foundation upon which decisions related to a facility can be made 
throughout its life cycle. The life cycle of a facility is defined as being from conception to demolition. The fundamental theory of BIM is collaboration by interested and concerned parties at various stages in the life cycle of a facility. BIM enables all collaborators to create, modify, view and otherwise extract information. BIM is promoted as being a shared digital representation. Information within BIM can be transmitted from computer to computer through exchange protocols which are based on open standards.

In their work [5] the authors explain the implication and utility of Building Information Modeling (BIM). Software companies such as Autodesk, Bentley and Graphsoft have developed BIM desktop applications that help construction engineering professionals to integrate and visual design and architecture. BIM reduces duplication of work while significantly boosting efficiency in terms of time and cost. As the authors explain, Jerry Laiserin, an industry analyst, introduced "Building Information Modeling" or BIM as it is commonly known, to describe construction, facilities management, and virtual design. BIM makes it possible to disseminate information within building industry through use of virtual models.

The authors in their work [6] discuss how BIM has gained tremendous attention in the field of architecture, engineering and construction (AEC). The authors have explained how cloud computing and service oriented architecture can significantly aid BIM to be more effective by reducing duplication, errors, and enhance productivity.

The work [7] of the authors discusses how cloud computing is relevant in the context of BIM and the construction industry. In the opinion of the authors, based on literature review and consultation with industry experts, cloud computing empowers construction industry to share data and process requirements in a cost-effective manner. The authors have outlined a governance model to promote development of research in the use of cloud computing by the construction industry.

In their paper [8] the authors have introduced a governance model using cloud computing for building data in a BIM environment.

Most authors have paid attention on creating frameworks which harness the potential of cloud computing with BIM. Our literature survey reveals that authors have focused on governance, establishing standards for exchange of data and information.

Today, one of the most popular suite of construction engineering tools, the Autodesk Revit 2016 family, support BIM applications over Citrix supporting working from remote locations using a single server, and Revit Server to support collaboration on shared Revit models across a wide-area network (WAN) [10].

\section{ISSUES AND CHALLENGES}

Our research and studies have revealed that the primary bottleneck in accessing mission critical applications and data from across diverse geographical locations is Internet bandwidth. In order to be effective and relevant, users should be able to work on applications that are hosted on cloud networks without losing out on quality and responsiveness of the applications. On the other hand, applications should also leave very little footprint on user devices so that the bulk of the processing can be done on servers hosted on cloud. Data storage and encryption should also be hosted on the cloud so that the users do not have to worry on losing out vital data in case of loss or damage to their personal devices.

While examining these factors, we realized that in order to minimize processing loads on user devices, our research direction needed to concentrate on video streaming. Applications can be streamed over the Internet as live video from cloud servers to user devices. The responses of the users are transmitted to servers where these are processed and responses streamed back as video frames.

\section{PROPOSED WORK}

We propose a secure cloud based data center managed by our Billboard Manager to delivery BIM applications over the Internet. We have incorporated Citrix XenApp 6.5 technology with HDX features. Citrix technology offers high quality streaming capabilities. In order to extend users a high definition video experience as far as possible over any network, we have proposed Citrix HDX as the underlying technology in our proposed model. Prominent commercial application developers have also adopted Citrix in order to deliver their BIM applications to end-users. To enable desktop virtualization scalable and cost-effective without being adversely affected by Internet and/or network bandwidth conditions, Citrix HDX tools include Citrix Independent Architecture (ICA).

A typical BIM system may include applications catering to energy simulation systems, HVAC simulations, air flow simulations, document management simulations, etc. Using our proposed model, a remote user would be able to connect easily to any of those applications by clicking on the respective published links. These links would be published by our proposed Billboard Manager [3]. Clicking on any of the links would connect a user to the appropriate BIM application.

The Billboard Manager (BM) will accept user requests from any appropriate device such as mobiles, laptops, or desktops. The BM will publish links of all available BIM applications currently online on its home page. As soon as a user clicks on any of these published links, the BM will connect the user to the BIM application session hosted on an available private cloud node server registered with it. In case a particular BIM application is not available on private cloud node servers, Billboard Manager will then switch on to the next suitable BIM application location that may be available in any of the public cloud nodes registered with Billboard Manager. BIM applications themselves can be installed on physical devices or virtual machines, and published through Citrix on each cloud node. The URL links to the BIM applications will be securely published on a SSL-based device.

In our proposed architecture, each BIM application will have its own URL registered with the Billboard Manager. The Billboard Manager will maintain an active table containing records of each of these links, response times to the respective cloud nodes, and status of last known activities.

A user is required to provide appropriate authentication details in the form of user name and password on the web page published by the Billboard Manager. Upon successful authentication, the user is connected through to the private cloud data center through a secure SSL VPN network tunnel. The links to the BIM applications is displayed on a web page. Each BIM application link connecting the respective remote desktop session is served by Citrix XenApp servers. To access the BIM applications, a user is required to click on the link and furnish authentication credentials appropriate and valid for the respective BIM application link. 
It is important to note that once a user sends in a request to connect to a given link, the Billboard Manager locates the most appropriate cloud node in terms of availability of BIM application sessions and response times. Once a user disconnects, or terminates a BIM session, a feedback is sent back from the user's device to the Billboard Manager notifying that the resource is free. The Billboard Manager tallies that response with that of the cloud node server serving the BIM application. Once termination is confirmed, the Billboard Manager updates its table accordingly, and this resource can then be made available to serve future user requests.

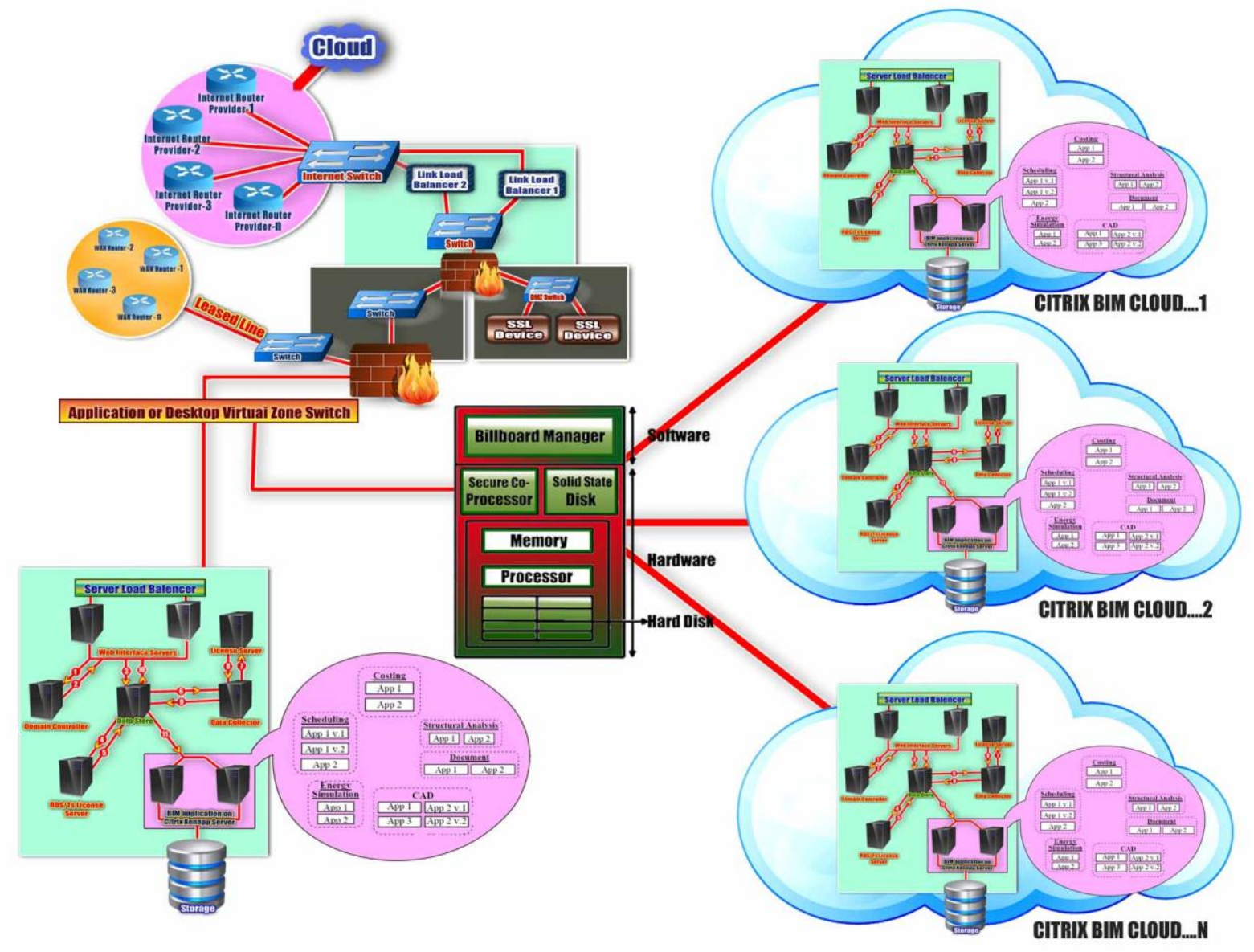

Figure 1: Proposed architecture using Billboard Manager to serve BIM applications over Internet in a cloud environment using Citrix technology.

The Billboard Manager will store encrypted data in locations after performing calculations with a set of parameters. Cryptographic co-processors aid in defining and implementing security protocols. A dedicated hardware set forms a Cryptographic co-processor which is able to handle tasks of either encryption or decryption [2]. Billboard Manager helps to select the appropriate storage node where encrypted data is deposited. As such, a major portion of the activities in our proposed system involves monitoring and communicating a large number of storage nodes by Billboard Manager. It is constantly aware of storage available on its own cloud network. Figure 1 shows the proposed architecture.

The architecture has been tailored keeping in mind the following.
Efficient storage of sensitive data in an encrypted form.
b. Manage, store and enable query of huge quantum of data.
c. Support for extremely precise access control.

Support extremely secure authentication
features.

Using the method suggested by us, it would be possible for an end-user to access applications running on remote desktop sessions over secure links. BIM applications would be instantly accessible and deployed over virtual platforms. The cloud service provider would provide a secure link, either over the Internet or via wireless networks, to the users.

\section{ALGORITHM}

Our proposed model consists of a three-stage algorithm as our projected Billboard Manager managed architecture takes care of managing and publishing active links, storage and encryption of data, presentation and authentication of user information. 


\section{STAGE 1: Data storage}

1. Billboard Manager stores information on cloud storage nodes registered with it. The data stored and maintained include those related to IP addresses, capacity, shortest node distance, storage space available, last accessed, and hardware details.

2. All the registered cloud nodes periodically send information to the Billboard Manager related to channel capacity and available storage space. The data varies from time to time and is also dependent on the location of the individual nodes.

3. At a point in time where $\mathrm{t}=0$, check whether storage capacity is not equal to zero.

4. If the above condition is satisfied, proceed to the next step. Else terminate loop. Billboard Manager makes appropriate notations in its records and notifies administrator.

5. Compare available storage capacities of those nodes which have storage greater than zero. The one with the greater storage is selected.

6. If the storage capacities are equal, choose the cloud node with the highest data rate. Otherwise go back to step 4.

7. Repeat steps 4 through 6 each time when choosing a new cloud storage node for handover.

8. Generate a list of the available cloud storage nodes and maintain a record of such with Billboard Manager.

9. Billboard Manager refreshes and updates a list of all available cloud storage nodes based on free space.

10. Billboard Manager compares the best fit cloud storage node to transmit data.

11. Connection is established with the best fit cloud storage node.

\section{STAGE 2: Citrix published links.}

1. Billboard Manager indexes all listed BIM applications published through Citrix.

2. It segregates and keeps tracks of all links and categorizes them as under private cloud or privatepublic (hybrid) cloud networks. Private cloud is considered to be also the local domain of the Billboard Manager.

3. The Billboard Manager constantly updates and refreshes the index that it maintains.

4. The Billboard Manager periodically assesses each of the published links and sorts them in the order of responsiveness and activity.

\section{STAGE 3: Home page and user authentication}

1. The Billboard Manager hosts the login page where users are expected to enter their user names and passwords.

2. The Billboard Manager checks for existence of users, user account validity; and also checks whether user has identified correctly.

3. In case a user does not have an account, the Billboard Manager redirects the user to an account creation page.

4. In the account creation page, the Billboard Manager collects the personal details of the user requesting creation of a new account, along with geographical location details, IP address, PC and/or mobile device specifications. These information are crucial for the Billboard Manager to make a close assessment on the type of link that may be served to the user, as well as identifying the nearest cloud nodes that can be used for serving the link, and storage of associated data.

5. In case of successful account authentication, the Billboard Manager presents a web page to the user detailing all available BIM applications ready for use.

6. The user is then allowed to select the BIM application that is needed to be used.

7. In case of identical BIM applications hosted through the Billboard Manager, the latter arranges the links in order as follows:

a. BIM application that is hosted on private cloud (or on local domain of the Billboard Manager) is listed at the very top.

b. BIM application that is hosted on a cloud node identified to be closest to the user's location.

c. BIM application hosted on a cloud node server that has longer response times, or higher latency.

d. BIM application that is currently in use and cannot be made available at that moment.

8. Once a user selects any of the links from among the published set hosted by the Billboard Manager, a link is established between the cloud node server running the BIM application on a Citrix server and the user.

9. The Billboard Manager automatically tracks the link that has been consumed by a user. The link is identified, and is displayed on the page as disabled.

10. As soon as a user exits a BIM application, the cloud node server sends a response to the Billboard Manager. The Billboard Manager updates its index, accordingly, and the link is set as active and ready to serve future requests.

\section{CONCLUSION}

Through our research, we have been able to observe and demonstrate how our proposed Billboard Manager can be deployed to actively support BIM applications through Citrix technology. We have also suggested the use of Billboard Manager to store, and offer encryption services, for data used in BIM applications. We have also explained why we are of the view that our proposed Billboard Manager based model can help in reducing processing loads on user devices through use of Citrix streaming technology. Our proposed model can help construction companies extend data processing capabilities to all stakeholders across diverse geographical locations. Using this model, commercial applications which are offered as Citrix-ready, can also harness cloud data storage facilities offered by Billboard Manager. The framework that we have discussed in this paper is aimed at offering scalability and offering optimum processing performance over the Internet and wireless networks. However, this is all accomplished without any significant rise in energy or time costs. This, in turn, is a step toward green computing and minimizing carbon footprint to a great extent.

\section{ACKNOWLEDGMENTS}

Authors gratefully acknowledge to CSE Department of University of Kalyani for providing lab and related facilities for do the research. 


\section{REFERENCES}

[1] Debabrata Sarddar, Rajesh Bose, Secure Co-processor and Billboard Manager Based Architecture Help to Protect \& Store the Citrix Xenserver Based Virtual Data, COMPUSOFT, An international journal of advanced computer technology, 3 (1), January-2014 (Volume-III, Issue-I).

[2] Praveen Ram C, Sreenivaasan G,Security as a Service (SasS),Securing User Data by Coprocessor and Distribut ing the Data,978- 1-4244-9008-0/10/\$26.00 @ 2010 IEEE.

[3] Debabrata Sarddar, Rajesh Bose, Creat ing a Secured Cloud Based Data Center Using Billboard Manager (BM) and Secuare Co-Processor, Internat ional Journal of Scient ific \& Engineering Research, Volume 4, Issue 12, December-2013.

[4] Frequently Asked Questions About the National BIM Standard-United States, http://www.nationalbimstandard.org/faq.php\#faq1

[5] Applying cloud computing technology to BIM visualization and manipulation, Tien-Hsiang Chuang1, Bo-Cing Lee1, and I-Chen Wu1*

[6] A critical review on new advancements in implemtation of it in construction industry: Integration of BIM with cloud computing Vasu Kathi1, Srinivas Vasam2, K. Jaganadha Rao3, M. V. Seshagiri Rao4

[7] Cloud BIM: Management of BIM data in a cloud computing environment T.H. Beach, PhD / Research Associate, beachth@cf.ac.uk Y. Rezgui, PhD / Director BRE Institute of Sustainable Engineering, rezguiy@cf.ac.uk O.F. Rana, PhD / Professor of Performance Engineering, o.f.rana@cs.cf.ac.uk Cardiff School of Engineering, Cardiff University, Wales, UK

[8] Cloud computing for the architecture, engineering \& construction sector: requirements, prototype \& experience Thomas H Beach1*, Omer F Rana2, Yacine Rezgui1 and Manish Parashar3

[9] System requirements for Autodesk Revit 2016 products http://knowledge.autodesk.com/support/revitproducts/troubleshooting/caas/sfdcarticles/sfdcarticles/Sy stem-requirements-for-Autodesk-Revit-2016products.html
[10] CAD Masters, Inc. To Offer Autodesk's 2012 Software Portfolio for Building Design, Infrastructure and Construction Industries.http://www.cadmasters.com/pressroom/2012_ Launch_PR.pdf

\section{AUTHORS PROFILE}

Rajesh Bose is currently pursuing Ph.D. from Kalyani University. $\mathrm{He}$ is an IT professional employed as Senior Project Engineer with Simplex Infrastructures Limited, Data Center, and Kolkata. He received his degree in M.Tech. in Mobile Communication and Networking from WBUT in 2007. He received his degree in B.E. in Computer Science and Engineering from BPUT in 2004. He has also several global certifications under his belt. These are CCNA, CCNPBCRAN, and CCA(Citrix Certified Administrator for Citrix Access Gateway 9 Enterprise Edition),CCA(Citrix Certified Administrator for Citrix Xen App 5 for Windows Server 2008).His research interests include cloud computing, wireless communication and networking.

Himadri Biswas, Assistant Professor in the Department of Computer Applications, Bengal College of Engineering \& Technology, Durgapur, West Bengal, INDIA. He has also 12 years of teaching experiences and worked under several institutions. He is currently pursuing Ph.D from University of Kalyani. He has completed his M. Tech in Computer Science \& Engineering from WBUT in 2010, and his MCA from St. Xavier's College, Kolkata under IGNOU, in 2004. His research interest includes cloud computing and mobile computing.

Debabrata Sarddar, Assistant Professor in the Department of Computer Science and Engineering, University of Kalyani, Kalyani, Nadia, West Bengal, INDIA. He has done Ph.D. He completed his M. Tech in Computer Science \& Engineering from DAVV, Indore in 2006, and his B.E in Computer Science \& Engineering from NIT, Durgapur in 2001. He has published more than 75 research papers in different journals and conferences. His research interest includes wireless and mobile system and WSN, Cloud computing. 